\title{
FEATURES OF COATED DIAMONDS FROM THE SNAP LAKE/KING LAKE KIMBERLITE DYKESYSTEM, SLAVE CRATON, CANADA, AS REVEALED BY OPTICAL TOPOGRAPHY
}

\author{
Alexander Yelisseyev ${ }^{1}$, Nikolai Pokhilenko ${ }^{1}$, Dmitry Zedginizov ${ }^{1}$, John Steeds $^{2}$, \\ ${ }^{1}$ Institute of Mineralogy \& Petrography, SB RAS, Russia; ${ }^{2}$ H.H.Wills Physics Laboratory, UK
}

\section{INTRODUCTION}

Coated diamonds are specific diamonds, which consist of a clear colorless core, often a well-formed octahedron of good quality, enveloped by a yellow, green or grey coat. The coat material is also diamond, but filled with dust-like matter, of which individual particles are generally sub-micron size and are not clearly resolvable with the optical microscope. Most of the recorded coated diamonds have been from the Congo where they form about $90 \%$ of the population (Kamiya and Lang, 1965), and Sierra Leone (up to 50\% in some deposits). They are very characteristic of Mbuji Mayi in Zaire, which is the world's largest producer of this type of diamond (Boyd et al, 1987). Coated diamonds have been found to be one of the main groups (up to $30 \%$ ) in the Canadian deposit under investigation: The systematic study of coated diamonds began in 1950 with a general description by Custers. Using X-ray topography Kamiya and Lang showed the coat has a fibrous structure interrupted by layers of fine particles. Later Lang (1974) concluded that the structural difference resulted from different growth mechanisms for the core and coat. The core grew continuously in the octahedral habit, by concentric, complete $\{111\}$ growth layers, while coat demonstrated a much rarer, abnormal mode when the growing crystal is broken up into a bundle of columns, growing outwards independently but with equal velocities. Faulkner et al (1965) found from an Electron Spin Resonance (ESR) study that the concentration of donor nitrogen (P1 centers in ESR or C-defects in optical spectroscopy) is low in coated diamonds: only about 30 ppm in the pieces cut from the coat. Angress and Smith (1965) found, according to IR spectroscopy, that considerable part of the nitrogen in the coat (up to 1700 $\mathrm{ppm}$ ) is in the A-form with dominating $1282 \mathrm{~cm}^{-1}$ peak. In the core nitrogen was found in A and/or B-form plus in many cases as platelets. There were some additional bands at 475, 1086 and $1105 \mathrm{~cm}$ in the IR spectra for the coat. A lot of additional lines, related to $\mathrm{OH}, \mathrm{HOH}$, $\mathrm{CH}, \mathrm{CO}_{2}, \mathrm{CO}_{3}{ }^{-}$and to silicate and other compounds in the numerous inclusions, were found in the coat related IR spectra of diamonds with strongly colored grey coating (Navon et al, 1988). The same authors also found that all inclusions are rich in various oxides such as $\mathrm{SiO}_{2}, \mathrm{~K}_{2} \mathrm{O}$.

Geochemical features and isotope characteristics of the Snap Lake/King Lake (SL/KL) kimberlites combined with results of the study of the crystalline inclusions in the diamonds suggest that their mantle sources were different from those for known kimberlites (Pokhilenko et al, 1997): The lithosphere beneath the Snap Lake area is abnormally thick (from 140 to at least $300 \mathrm{~km}$ ). This means that the pressure interval of the natural diamond formation lithosphere was from 37 to at least 110 kbar while the normal pressure interval is from 37 to $\sim 65$ kbar. As a result differences in the internal structure and physical characteristics of the Snap Lake diamonds, formed at abnormally high pressures, would be expected.

In the present paper the similarity between coated diamonds from SL/KL and other deposits (Zaire etc) is demonstrated using microscopic study in transmitted light, birefringence, photoluminescence (PL) and X-ray topography. New information about types and spatial distribution of existing point defects and resulting changes in optical properties was obtained using local absorption and confocal laser-excited PL spectroscopy . An attempt to reconstruct the growth history of coated diamonds was undertaken.

\section{EXPERIMENTAL}

Two typical rounded diamonds, (SL-00/31 and SL$00 / 14)$ were prepared as $\{110\}$ plates, about $0.5 \mathrm{~mm}$ thick, for detailed studies using all available techniques. Transmission, birefringence, X-ray and PL images were obtained and maps of $\mathrm{H} 3$ emission and Raman light scattering were built. Transmission and birefringence images were obtained in visible spectrum using a 100 $\mathrm{W}$ incandescent lamp; PL images were obtained using $366 \mathrm{~nm} \mathrm{Hg}$ line for excitation and $488 \mathrm{~nm}$ line of $\mathrm{Ar}^{+}$ laser was used for mapping. The coating of the two diamonds was either a well-pronounced yellow color (Fig.1) or only slightly yellowish. Coloration is usually much stronger near the core/coating boundary (Fig.1A, 2A). The inclusion density and the intensity of the resulting grey color were much lower than for samples of Navon et al, (1988). A Bruker IFS 113 FTIR 
spectrometer combined with optical microscope was used to measure the IR absorption spectra with a $2 \mathrm{~cm}^{-1}$ resolution at ro om temperature for a set of points along specific lines across the sample. The typical diameter of the light spot was 50 microns with 50 to 150 microns distance between points. In the one-phonon region, spectra were divided into individual components related to A and B- centers and the nitrogen concentration was calculated based on relations determined by Woods et al, (1990) and Boyd et al, (1995). The PL spectra were recorded using the Renishaw micro-Raman spectrometers fitted with an Oxford Instruments Microstats. When measuring spectra the $325 \mathrm{~nm}$ line of a HeCd laser, and the 488/514 nm lines of an $\mathrm{Ar}^{+}$laser were used for excitation. Spectra were recorded for either a set of points distributed along the sample diameter or forming a net covering the whole sample. In the latter PL or Raman maps were built using special Renishaw software. The photoluminescence excitation spectra (PLE) were measured using a $1 \mathrm{~kW}$ Xe lamp with diffraction MDR2 monochromator for separation of excitation spectral range, and the MDR1 double diffraction monochromator for monitoring the PL emission.

\section{RESULTS}

\section{PATTERnS}

Both, SL-00/31 and SL-00/14, have high quality colorless crystals of octahedral habit as a core: surrounded by a well-pronounced yellow translucent coating about $0.5 \mathrm{~mm}$ thick (Figs.1A, 2A). The coating is better revealed when using blue light for examination (Fig.2A). In Xray topograms the fibrous structure of the coat is well pronounced with strong strains in the lattice being revealed with crossed polarizers (Fig.2B). Birefringence is relatively weak in the core produced by cracks and inclusions if they are present (Fig.1C, 2B). Sometimes the stress field extends from coating to the core. Birefringence is particularly strong in the coating because of its fibrous structure and variety of boundaries between single crystal needles. There is a thin and relatively sharp boundary, (less than 10 microns thick) formed by inclusions, between the core and the coating. Large dark spots in Figs 1A, 2A correspond to cracks. The coating can be completely yellow in color (SL-00/31 Fig.1A) or almost colorless with a yellowish layer at the boundary between core and coating (SL-00/14, Fig.2A). The PL patterns show that both core and coat are not homogeneous: there is an outer nonluminescent layer in the core and layers with different PL color in the coat (Fig.2C, D). The PL

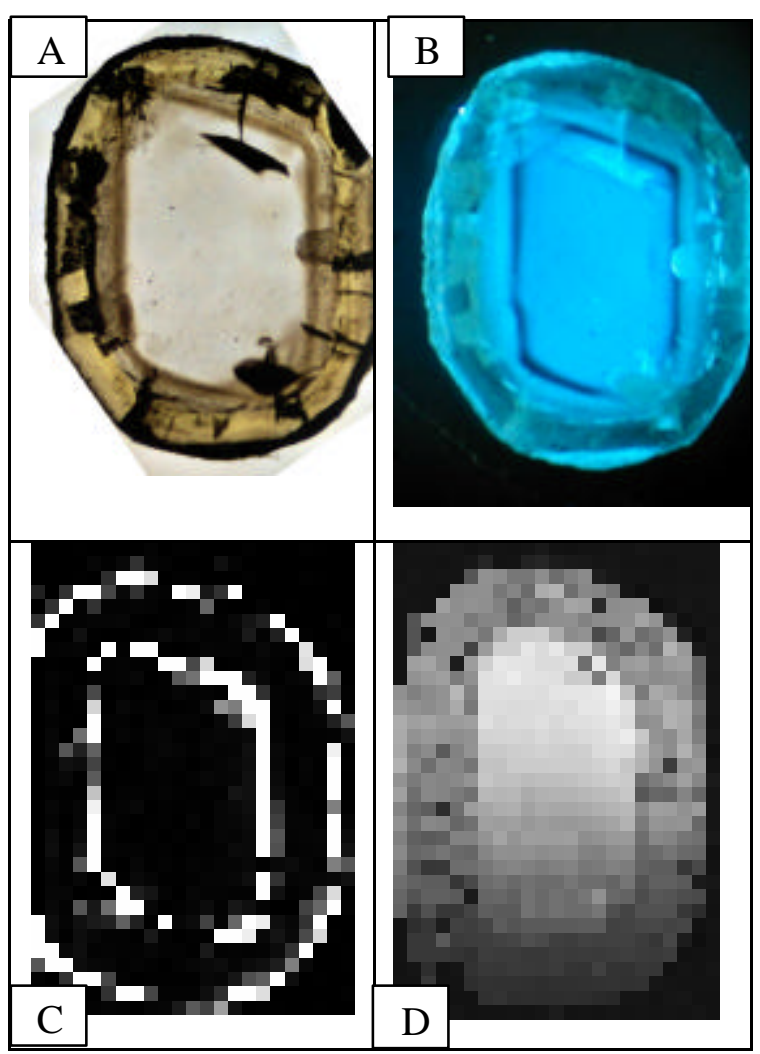

Fig.1 Photographs of coated SL-00/31 natural diamond, obtained in transmitted nonpolarized light (A) and PL pattern at UV excitation (B). Results of mapping with a 100 ? $\mathrm{m}$ step for $\mathrm{H} 3$ emission in PL and Raman scattering are given in (C) and (D), respectively

was particularly intense for SL-00/14: blue in the core, yellow-green coating and the near-surface PL varying from blue to white. The thickness of the nonluminescent layer varies from $\sim 10$ microns in SL00/31 to 200-250 microns for SL-00/14. A slightly pronounced striation of this area, with the straight lines parallel to the core [111] faces, can be seen. The yellowish coating formed over this nonluminsecent area does not repeat the octahedral shape of the core striation but is of a rounded shape. This suggests that there was a stage of dissolution of the original octahedron. For SL-00/31 the maps of H3 emission and Raman light scattering intensity, (obtained at $488 \mathrm{~nm}$ excitation from $\mathrm{Ar}^{+}$laser and 100 microns scanning steps) are shown in Fig 1C and 1D. The maximum intensity of the $\mathrm{H} 3$ luminescence occurs at the core/coating boundary and near the crystal surface. The Raman scattering is stronger in the core and somewhat weaker in the coating. The poor contrast in the lower part of the pattern in Fig.1D is due to some deviation from the horizontal position for the sample with resulting defocusing of the exciting laser beam of the confocal system. 


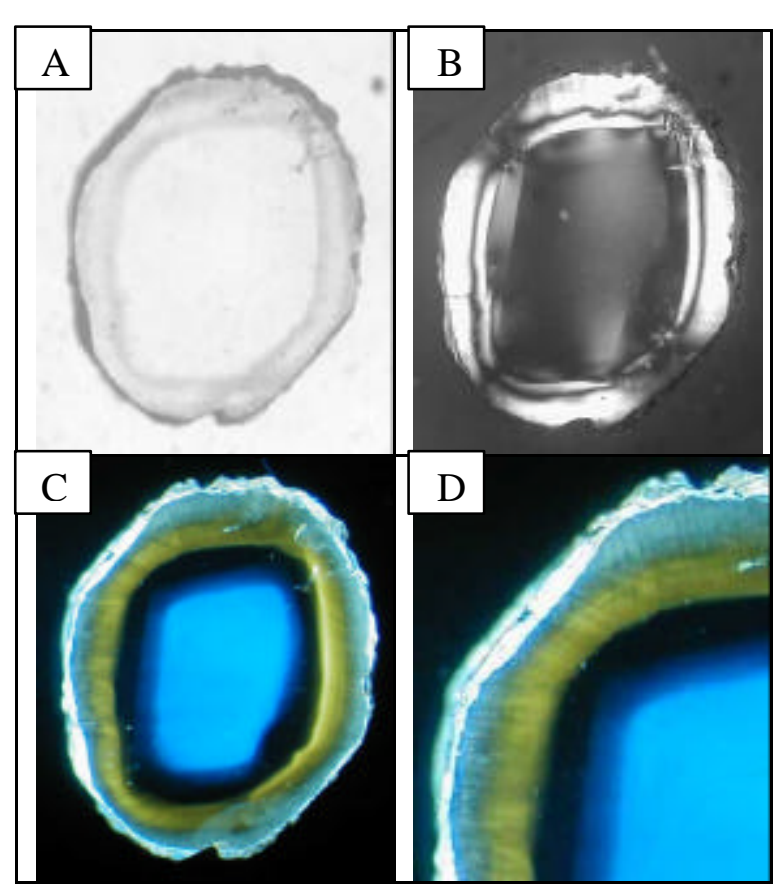

Figure 2. Photographs of coated SL-00/14 natural diamond, obtained in transmitted blue light (A) light or through crossed polarizers (B). The PL pattern at UV excitation (C) with a detail (D), demonstrating a fibrous structure of the coating are shown.

\section{ABSORPTION SPECTROSCOPY}

The IR spectroscopy shows only nitrogen complexes such as A and B-defects are typical of coated diamonds (Fig. 3). In the core, B-defects dominate with some input from the A-defects, whereas in the coat nitrogen is mainly in the A-form. The total nitrogen concentration is approximately constant $(\sim 1400$ to 1700 ppm) across SL-00/31. Platelets B' is also a typical defect but they are located in the core only. Lines 1405 , $3107 \mathrm{~cm}^{-1}$ related to $\mathrm{C}-\mathrm{H}$ bonds were found only in the coat, their maximum intensity occurring in the internal layer of the coat. There are some variations in concentration/state of nitrogen in the coat, which confirms its multilayer structure and coincides with results of the preliminary visual examination. For diamond SL-00/14 the same features were observed, but the nitrogen was completely in the Bform in the core and in the A-form in the coat. The nitrogen content in the coat was about $2000 \mathrm{ppm}$ and about three times lower in the core, with a low nitrogen layer ( $<20 \mathrm{ppm})$ between them The short-wave edge of the transparency region is determined by A-defects: crystals are transparent at wavelength $>270 \mathrm{~nm}$ with narrow lines at $306,316 \mathrm{~nm}$ plus N3 system with zero-phonon line (ZPL) at $415 \mathrm{~nm}$. In the coat, crystal becomes

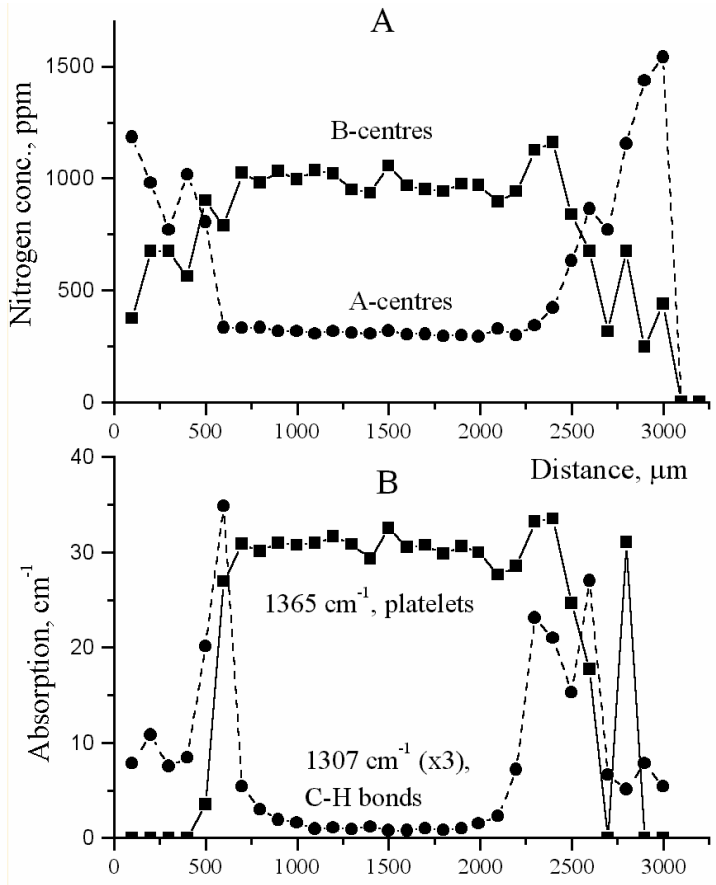

Figure 3 Spatial distribution of the main defects across sample SL-00/31 according to IR absorption spectroscopy.

transparent at $\sim 320 \mathrm{~nm}$ with a broad with a broad structureless shoulder beginning from $600 \mathrm{~nm}$. In the nitrogen-free layer, which is particularly wide in SL$00 / 14$, the fundamental absorption edge is at $225 \mathrm{~nm}$ with 230, $236 \mathrm{~nm}$ bands of the N9 system, related to Bdefects.

\section{PHOtOLUminesCenCe}

Three PL spectra, recorded at the UV excitation at three specific points 1-3 (near surface, in the coat and in the core, respectively) of SL-00/31, are shown in Fig.4. In the core N3 with specific vibronic structure is dominating (Fig.5A), whereas in the coat a broad structureless band in the same spectral region can be seen. This is considered to be due to the dislocation Aband well known both for synthetic and natural diamonds (Zaitsev, 1999). The other broad band centered at $550 \mathrm{~nm}$ has an envelope similar to that for the sum of vibronic systems with ZPLs at 488.9 and $523.2 \mathrm{~nm}$ typical of cube-shaped natural diamonds (Fig.4, curve 4) and associated with the nickel-nitrogen complexes (Nadolinny and Yelisseyev, 1993). This band is relatively weak in SL-00/31, but is responsible for the intense yellow-green PL in SL-00/14 (Fig.2, C and D). There are other centers such as H3, S1 with a similar envelope but their absorption spectra are a mirror image of the PL. As a result, this emission can be excited only using visible light. Indeed, the photoluminescence excitation spectrum (PLE) recorded 


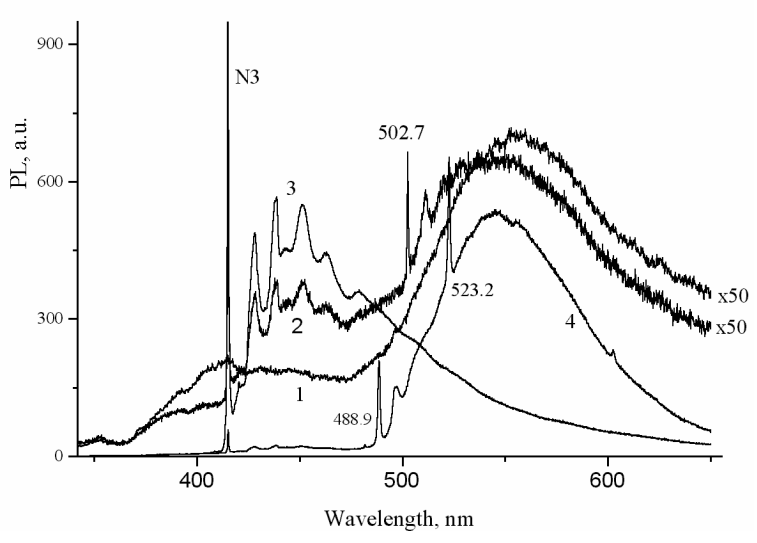

Fig.4 The PL spectra, recorded for SL-00/31 at $325 \mathrm{~nm}$ excitation for points located 50 ? $\mathrm{m}$ (curve 1), 500 (2) and 850 ? m (3) from the surface. Spectrum 4 shows PL due to nickelnitrogen complexes in SL-00/47 (for comparison).
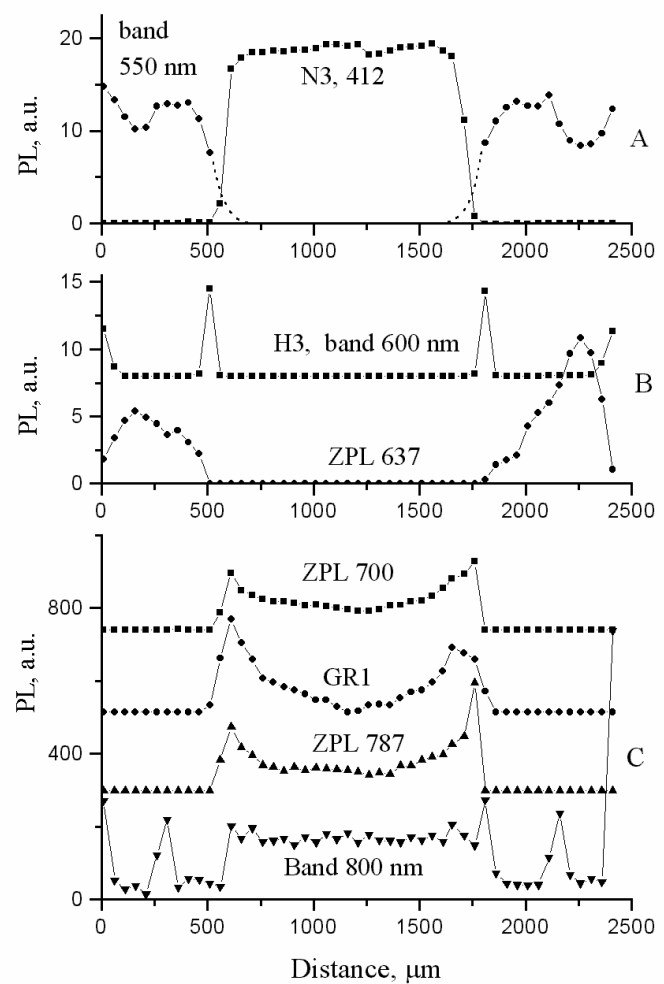

Fig. 5 Spatial distribution of main features in PL spectra of SL-00/31 diamond at 325 (A), 488 (B) and $514 \mathrm{~nm}$ (C) excitation. $\mathrm{T}=8 \mathrm{~K}$.

directly for the yellow-green PL in the coat demonstrates two well-pronounced bands centered at 320 and $430 \mathrm{~nm}$ (Fig.6, curve 2), which are similar to beta- and alpha-bands in the PLE spectra of NE1-NE3 nickel-nitrogen complexes, respectively (Nadolinny and Yeliseeyev, 1993). A lot of other systems (ZPLs etc)

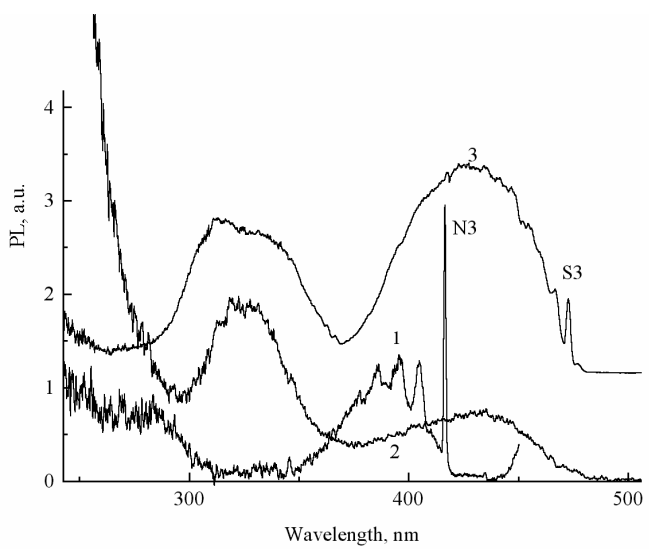

Fig.6 The PLE spectra for the blue PL in the core (1) and for yellow-green PL in the coat (2) for SL-00/14 diamond, recorded at $80 \mathrm{~K}$. Spectrum 3: the PLE related to Ni-N complexes in synthetic S727 diamond, which has been annealed at 2000 ?C, it is shifted upwards for clarity.

are excited when using 488 and $514 \mathrm{~nm}$ lines of $\mathrm{Ar}^{+}$ laser. In this study attention is paid only to those with known structure, their spatial distribution is given in Fig.5, B and C. Here GR1 is a neutral single vacancy $\mathrm{V}^{0}$ whereas 637 and $\mathrm{H} 3$ (with $503.2 \mathrm{~nm}$ ZPL) systems are believed to be due to vacancy-nitrogen complexes as NV and NVN, respectively (Zaitsev, 1999). The 700 and $787 \mathrm{~nm}$ ZPL are typical of synthetic nitrogen-rich diamonds grown in the $\mathrm{Ni}$-containing system, which were afterwards annealed at temperatures $\mathrm{T}>1700 \mathrm{C}$ : thus the defects are related to nickel-nitrogen complexes (Yelisseyev et al, 2002).

The main results of the analysis of the defect spatial distribution in coated diamonds are:

1. Coated diamonds are a result of a multistage growth process with stages separated in time. The core of octahedral habit grew by layer-on-layer mechanism. The coating demonstrates the well-pronounced fibrous structure related to "abnormal" crystal growth of higher growth rates when a lot of impurities such as nitrogen, nickel, hydrogen etc were encapsulated. The rounded shape of the coated diamonds is due to combined effect of the intermediate partial solution and fibrous growth at the last stage of diamond formation;

2 . The internal portion of the core contains nitrogen with a high degree of aggregation (N3, B-centers) and platelets, the outer part of the core usually being depleted in nitrogen (close to IIa type) The A-defects dominate in the coating.

3. Intense H3 luminescence was found in a thin layer coincident with the maximum inclusion concentration, as well as near the surface. Analysis of the ZPL fine structure reveals strains of about $0.3 \mathrm{GPa}$ around the $\mathrm{H} 3$ centers in the core. 
4. Vibronic systems related to the $\mathrm{Ni}-\mathrm{N}$ complexes were found both in the core (weak ZPLs) and in the coat. In the core the systems with well pronounced 700 and $787 \mathrm{~nm}$ ZPLs are typical of natural and synthetic diamonds from the Ni-containing system after annealing at $\mathrm{T}>1700 \mathrm{C}$. In the coat the structureless 550 $\mathrm{nm}$ band, responsible for intense yellow-green PL, is identified as a sum of the vibronic systems associated with NE2 and NE3 paramagnetic defects. Identification was made when the envelopes of the PL and PLE spectra were analyzed. The combined ESR and optical study showed that these systems are due to isomers of the defects where nickel ion in divacancy position surrounded by three nitrogen atoms (Nadolinny et al, 1993). A study of synthetic diamonds, annealed at different temperatures, showed that 700 and $787 \mathrm{~nm}$ systems appear at higher temperatures and thus can be related to $\mathrm{Ni}-\mathrm{N}$ complexes with higher aggregation degree $(\mathrm{n}>3)$ (Yelisseyev et al, 2002).

5. The H3 defects in the internal layer saturated with inclusions are likely to be formed directly during growth. Immediately after their growth the diamond coat contained $\mathrm{N}$ mainly as single atoms (C-centers), and some vacancies appeared as a result of irradiation with fast electrons or gamma-rays. During further annealing the vacancies became mobile at $\mathrm{T} \sim 400 \mathrm{C}$, reached C-centers and formed $\mathrm{NV}$ defects responsible for the 637 system. The presence of A-and B-defects shows that afterwards there was a stage of annealing at higher temperature $(\mathrm{T}>1500 \mathrm{C})$ when nitrogen atoms became mobile and formed the A and B-defects. In the coat this temperature could be lowered and the aggregation process could be considerably accelerated by transition metal ions e.g. nickel (Fisher and Lawson, 1998), which has a particularly high concentration in the coat. High $\mathrm{H} 3$ concentration in the near surface layer is supposed to be a result of irradiation by heavy particles (alpha-particles) with small penetration depth and subsequent annealing

\section{ACKNOWLEDGEMENTS}

This work was supported by Grants No 98-05-65283 and 02-05-65075 from the Russian Foundation for Basic Research. A.P.Y. thanks the Royal Society for the Vis iting Research Fellowship.

\section{REFERENCES}

Angress, JF and Smith, SD (1965): The observation of defectactivated one-phonon infra-red absorption in diamond coat, Phil.Mag., 12, pp. 415-417.

Boyd, SR, Mattey DP, Pillinger, CT, Milledge, HJ, Mendelssohn, M, and Seat, M (1987): Multiple growth events during diamond genesis: an integrated study of carbon and nitrogen isotopes and nitrogen aggregation state in coated stones, Earth \& Planetary Science Letters, 86, pp. 341-357.

Boyd, SR, Kiflawi, I, Woods, GS (1995): Infrared absorption by the $\mathrm{B}$ nitrogen aggregate in diamond, Philosophical Magazine B, 72, pp. 351- 361 .

Custers, JFH, (1950): Amer.Miner.35, pp.51-58.

Faulkner, EA, Whippey, PW, Newman, RC (1965): Electron spin resonance in diamond coat, Phil.Mag., 12, pp. 413-414.

Fisher, D, and Lawson,S, (1998): The effect of nickel and cobalt on the aggregation of nitrogen in diamond, Diam.Rel. Mat., 7, pp. 299-304.

Kamiya, Y and Lang, AR, (1965): On the structure of coated diamonds, Phil.Mag. 11, pp 347-356.

Lang AR, (1974): Glimpses into the growth history of natural diamonds, J.Cryst.Growth, 24/25 pp.108-115.

Nadolinny, VA, and Yelisseyev, AP (1993): New paramagnetic nickercontaining centers in diamond, Diamond \& Rel.Mat., 3 pp.17-22.

Navon, O, Hutcheon, ID, Rossman, Grand Wasserburg, GJ (1988): Mantle-derived fluids in diamond microinclusions, Nature, 335, pp. 784-789.

Pokhilenko, NP, McDonald, JA, Hall, AE and Sobolev, NV, (2001): Abnormally thick Cambrian lithosphere of the South-East Slave Craton: evidence from crystalline inclusions in diamonds and pyrope composition in Snap Lake Kimberlites, The Slave KAAPVAAL Workshop, Merrickville, Ontario, Canada, pp.49-51.

Woods, GS, Purser, GC, Mtimculu, ASS and Collins, AT (1990): The nitrogen content of type Ia natural diamonds, J.Phys.Chem.Solids, 51, pp. 1191-1197.

Yelisseyev, AP, Nadolinny, VA, Feigelson, BN, Babich Yu.V. (2002): Spectroscopic features due to Nirelated defects in HPHT synthetic diamonds, Int.J.of Modern Phys.,B, Condensed Matter Physics, 16, pp. 900-905.

Zaitsev, AM, Optical properties of diamond, Ruhr-University of Bochum, 1999, p.320.

Contact: AP Yelisseyev, IMP SB RAS, 3 Ac.Koptyug Av.,

Novosibirsk630090 Russia. E-mail:elis@ mail.nsk.ru 